\title{
Effects of non-uniform nanoparticle concentration on entropy generation
}

\author{
A. Mehmood ${ }^{a}$, S. Khan ${ }^{a, b}$, and M. Usman ${ }^{c}$ \\ ${ }^{a}$ Department of Mathematics and Statistics, \\ International Islamic University Islamabad, 44000 Pakistan. \\ e-mail: ahmerqau@yahoo.co.uk; sajidkhanqau@yahoo.com; muhammadusman@vu.edu.pk \\ ${ }^{b}$ Department of Mathematics, University of Wah, Wah Cantt, 47040 Pakistan. \\ ${ }^{c}$ Department of Mathematics, Virtual University of Pakistan.
}

Received 19 February 2021; accepted 21 July 2021

\begin{abstract}
The entropy generation analysis of a thermal process is capable of determining the efficiency of that process and is therefore helpful to optimize the thermal system operating under various conditions. There are several ingredients upon which the phenomenon of entropy generation can depend, such as the nature of flow and the fluid, the assumed conditions, and the material properties of the working fluid. However, the dependence of entropy generation phenomenon upon such properties has so far not been fully realized, in view of the existing literature. On the other hand, based upon the existing studies, it has been established that the non-uniform concentration of nanoparticles in the base fluid does cause to enhance the heat transfer rate. Therefore, it is logical to investigate the entropy production under the impact of non-homogenous distribution of nanoparticles. Based upon this fact the aim of current study is to explore a comprehensive detail about the influence of non-homogeneous nanoparticles concentration on entropy production phenomenon by considering a laminar viscous flow past a moving continuous flat plate. Non-uniform concentration is considered in the nanofluid modeling in which the Brownian and thermophoretic diffusions are considered which impart significant effects on velocity and temperature profiles. An exact self-similar solution to this problem is observed to be possible and is reported. The effects of various controlling physical parameters such as Brinkman number, Schmidt number, Prandtl number, diffusion parameter, and concentration parameter on both local as well as total entropy generation number and Bejan number are elaborated by several plots and tables. The obtained results reveal a significant impact of all aforementioned parameters on entropy generation characteristics. It is observed that by a $20 \%$ increase in nanoparticles concentration the total entropy generation is increased up to $67 \%$ for a set of fixed values of remaining parameters.
\end{abstract}

Keywords: Nanofluid; non-homogeneous modeling; entropy generation; moving surface.

DOI: https://doi.org/10.31349/RevMexFis.68.010601

\section{Introduction}

The efficiency and optimum design of heat and mass transferring engineering systems can be analyzed with the aid of second law of thermodynamics. According to this law, in an irreversible process the entropy of thermal system grows, which ultimately affects the efficiency of the whole system. This means that one can further improve the thermal efficiency of any heat exchanger by minimizing the entropy generation. For this purpose, the understanding of entropy generation phenomenon and the realization of its dependence upon other ingredients had been a fundamental topic of interest in heat transfer science. The reason behind this fact is that, in the heat transfer processes temperature and viscosity of the fluid are the key sources of entropy production [1-4]. Owing to these facts, most of the researchers directly look forward to improve the productivity of a thermal processes by the minimization of entropy generation. This gave rise to theoretical studies where the authors use to consider variety of flow assumptions for the investigation of entropy generation phenomenon. For instance, Yilbas [5] examined the entropy generation in an annulus of concentric cylinders with a moving outer cylinder. He found that the entropy generation minimizes as the width of annulus decreases. Mahmud and Fraser [6] investigated the second law of thermodynamics for forced convection flow inside a channel. Butt et al. [7] discussed the contribution of thermal radiation in the analysis of entropy generation in classical Blasius flow. Makinde [8] discussed the irreversibility phenomenon for variable viscosity with the influence of Newtonian heating. He observed that a decrease in entropy production occurs with an increment of variable viscosity. Butt et al. [9] determined that, the entropy generation in a thermal system can be reduced due to the presence of hydrodynamic slip. Recently, Mehmood et al. [10] discussed the impact of wavy surface texture on entropy generation phenomenon. The use of nanoparticles in regular fluid has a wide range of applications in various fields of engineering such as microsystem cooling, nano-medicine, and energy convection. In order to achieve the efficiency of thermal engineering systems (in the perspective of design and operation), the system should be designed in such a way that it maximizes the heat transfer and minimizes the entropy generation. Many authors discussed the entropy generation phenomenon by considering different geometries and flow assumptions of heat transfer in nanofluid. Rashidi et al. [11] investigated the irreversibility phenomenon by considering a magnetic field in the presence of uniform distribution of three different types of nanoparticles. They observed that in the swirling disk flow, the en- 
tropy generation minimizes by decreasing values of magnetic parameter. Noghrehabad et al. [12] discussed the nanofluid flow along the stretching surface by considering heat generation phenomenon. Their numerical results showed that an increase in heat generation parameter leads to the minimization of entropy production. Rashidi et al. [13] discussed the entropy production phenomenon for MHD blood flow in the presence of nanoparticles. Significant effects upon entropy production were observed in the main flow region with the enhancement of Brinkman number. Almakki et al. [14] studies the entropy generation in MHD nanofluid flow along a non-linearly stretching surface. They concluded that entropy generation is more affected by viscous dissipation in case of large values of Reynolds number. However, all the aforementioned and a lot of other similar studies available in literature rely on the fact that the distribution of nanoparticles in the base fluid is constant/uniform. But, actually, reality is quite different. In general, the nanoparticles' distribution in the base fluid is non-uniform. This assumption gives rise to several difficulties in the mathematical handling of such problems. To avoid such difficulties, usually a homogenous model is preferred. However, studies revealed that the assumption of homogenous distribution of nanoparticles in a nanofluid leads to certain incorrect results. For instance, Magia [15], Avramenko et al. [16-17], and Mehmood and Usman [18] showed that the rate of heat transfer is underpredicted by considering the homogeneous distribution of nanoparticles. Furthermore, experimental study of Frank et al. [19] also shows that the distribution of nanoparticles in a nanofluid is non-uniform in nature. Migration of nanoparticles is observed through confocal microcopy view. The experimental results showed that, an increment in migration rate of nanoparticles leads to an augmentation in convective flow rate. Non-uniform suspension of nanoparticles in a nanofluid flow between rotating plates was also considered by Mehri et al. [20]. Ding and Wen [21] discussed the migration of nanoparticles in a pipe flow and found that the concentration of nanoparticles in the central region of the pipe is greater than the wall region. They found that the viscosity gradient and Brownian motion are responsible for non-uniform distribution of nanoparticles. Avramenko et al. [16] obtained a self-similar solution of nanofluid flow with the consideration of nonhomogenous nanofluid modelling. They observed that due to the non-uniform distribution of nanoparticles, the increments in heat and mass transfer rates are more pronounced. Recently, Mehmood and Usman [18] explored the non-uniform concentration effects on moving plate boundary layer. They compared the percent increments in heat transfer rate due to homogenous and non-homogenous nanoparticles concentration models. They concluded that the non-homogenous distribution of nanoparticles leads to a substantial increase in heat transfer rate in comparison to homogenous distribution. These astonishing results of increased heat transfer rate for a non-homogenous distribution of nanoparticles are due to various possible factors which explain the relative slipping effects of nanoparticle inside the regular fluid. These key factors are very well explained by Buongiorno [22] where he gave their mathematical form which enables one to incorporate the non-uniform distribution effects of nanoparticle in the fluid flow and heat transfer problems. The Brownian motion phenomena is the first factor which highlights the role of stochastic motion of nanoparticles within the fluid. The remaining factors are thermophores, and diffusiophores which are responsible for the nanoparticle's diffusion owing to the temperature and concentration gradient. Furthermore, for the nanoparticle's concentration, a transport equation was also taken into account along with transport equations of fluid flow and heat transfer. The details of all these mechanisms are further given in the mathematical description of the selected non-homogenous model. From above cited studies (related to entropy production in nanofluid) and to the best of the authors' knowledge the entropy production phenomenon had only been discussed for uniform distribution of nanoparticles so far. Being convinced from the literature review in the previous paragraph that the non-uniform distribution of nanoparticle seems to be more effective as compared to uniform distribution, the present study aims to extend the work of Mehmood and Usman [18] by considering the entropy production phenomenon in a boundary-layer flow of a nanofluid involving non-homogenous modelling of nanofluid. It is interesting to observe the role of variable nanoparticle concentration on entropy production. Because of the variable nature of nanoparticles concentration, the averages of entropy generation and Bejan number are computed.

\section{Problem statement and self-similar formu- lation}

A steady, two-dimensional boundary layer flow of an incompressible nanofluid due to a moving continuous surface is considered. A schematic diagram of flow geometry is shown in Fig. 1. The governing equations of this flow are the famous laws of conservations of mass, momentum, energy, and concentration. The consideration of a nanofluid flow, then, requires appropriate modifications in the said governing laws. However, there exists no unique model for the nanofluids rather a variety of empirical and semi-empirical models is available in literature. Moreover, complete information about

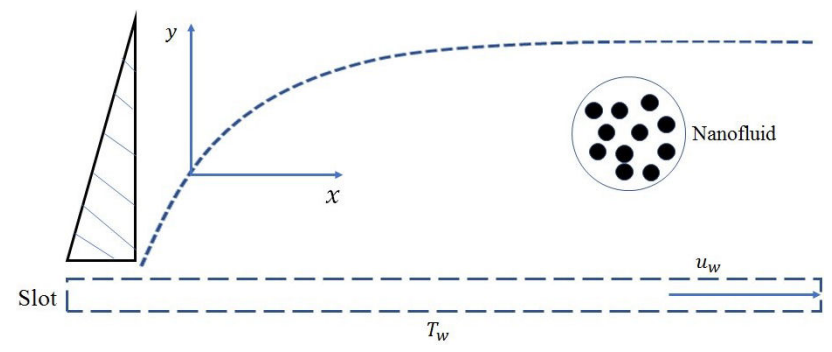

FIGURE 1. Physical model of the problem. 
the correct physical reasons about the significant enhancement of heat transfer anomaly is not yet available. However, based upon their observations several researchers reported different reasons. Some of the important contributors (such as [25]) concluded that the dispersion of nanoparticles and hence the enhanced convective mixing (turbulence) are the primary agents behind an enhanced rate of heat transfer. Later on Buongiorno [22] proved and reported that the said two agents are not fully responsible for an enhanced heat transfer process of nanofluid, rather their contribution in this regard is negligible. To identify the correct reasons, he considered the possible transport mechanisms: thermophoresis, inertia, Brownian diffusion, diffusio-phoresis, Magnus effects, gravity effects, etc. and reported that only the Brownian motion and the thermophoresis are significantly important contributors towards the expedition of heat transfer process. Based on these observations he improved the laws of conservation of mass, momentum, energy, and concentration in the following way:

$$
\begin{aligned}
\nabla \cdot(\rho v) & =0 \\
\left(\frac{\partial}{\partial t}+(v \cdot \nabla)\right) \rho v & =-\nabla p+\nabla \cdot\left(\mu\left(\nabla v+\nabla v^{T}-\frac{2}{3} \delta \nabla \cdot v\right)\right), \\
\left(\frac{\partial h}{\partial t}+(v \cdot \nabla)\right) \rho h & =\nabla \cdot(k \nabla T)+\rho_{p} c_{p}\left(D_{B} \nabla \phi \cdot \nabla T+D_{T} \frac{\nabla T \cdot \nabla T}{T}\right), \\
\rho_{p}\left(\frac{\partial \phi}{\partial t}+(v \cdot \nabla) \phi\right) & =\nabla \cdot\left(\rho_{p} D_{B} \nabla \phi+\rho_{p} D_{T} \frac{\nabla T}{T}\right),
\end{aligned}
$$

where $v$ is the velocity vector, $h(=c T)$ is enthalpy in which $c$ and $T$ represent specific heat and temperature, respectively, and $\phi$ denotes the concentration of nanoparticles. The above transport equations have been written for an incompressible viscous flow involving no body force and no chemical reaction. The nanofluid is assumed to be a dilute mixture of nanoparticles and base fluid where both are in thermal equilibrium. The thermal transport process involves negligible effects of viscous dissipation and radiative heat transfer. Because of the incompressibility conditions the fluid density is assumed to be spatially independent but essentially an appropriate function of volume fraction $\phi$ and hence an indirect dependent on space. Such an indirect dependence of $\rho$ on $\phi$ and the dependence of $\phi$ on space thus consequently make this model a non-homogeneous in nature, with regard to the nanoparticle concentration. Though, there exist several such models (such as [22,23]) which do not involve the variation of nanoparticles volume fraction and are commonly known as homogeneous models. Although such a homogenous modelling results in a great mathematical simplicity and ease of handling, it significantly under-predicts the rate of heat transfer. In this study, this has been shown that the non-homogeneous model gives further enhanced rate of heat transfer. Based on similar arguments the involved fluid properties in system (1)-(4) are defined as follows:

$$
\begin{aligned}
\mu & =\frac{\mu_{f}}{(1-\phi)^{2.5}}, \quad D_{B}=\frac{k_{B} T}{3 \pi \mu d_{p}}, D_{T}=\beta \nu \phi, \\
\rho & =(1-\phi) \rho_{f}+\phi \rho_{p}, \quad \rho c=(1-\phi)(c p)_{f}+\phi(c p)_{p}, \quad \beta=(1-\phi) \beta_{f}+\phi \beta_{p}, \\
K & =k_{f}\left[\frac{k_{p}+2 k_{f}+2 \phi\left(k_{p}+2 k_{f}\right)}{k_{p}+2 k_{f}-\phi\left(k_{p}+2 k_{f}\right)}\right],
\end{aligned}
$$

where the dependence of these quantities on $\phi$ is quite obvious. Here $k_{B}$ and $d_{p}$ are the Boltzman constant and diameter of particles, respectively. The subscript $f$ stands for fluid and $p$ stands for particles. Equations (1)-(4), after the application of boundary layer approximation take the following form (see for instance [16,18]):

$$
\begin{aligned}
\frac{\partial(\rho u)}{\partial x}+\frac{\partial(\rho v)}{\partial y} & =0, \\
u \frac{\partial(\rho u)}{\partial x}+v \frac{\partial(\rho u)}{\partial y} & =\frac{\partial}{\partial y}\left(\mu \frac{\partial u}{\partial y}\right), \\
u \frac{\partial(\rho h)}{\partial x}+v \frac{\partial(\rho h)}{\partial y} & =\frac{\partial}{\partial y}\left(k \frac{\partial T}{\partial y}\right)+\rho_{p} c_{p}\left(D_{B} \frac{\partial \phi}{\partial y} \frac{\partial T}{\partial y}+\frac{D_{T}}{T}\left(\frac{\partial T}{\partial y}\right)^{2}\right), \\
u \frac{\partial \phi}{\partial x}+v \frac{\partial \phi}{\partial y} & =\frac{\partial}{\partial y}\left(D_{B} \frac{\partial \phi}{\partial y}+\frac{D_{T}}{T} \frac{\partial T}{\partial y}\right),
\end{aligned}
$$

where $u$ and $v$ represent the velocity components along $x-$ and $y-$ axes, respectively. The appropriate boundary conditions of 
this flow are given by

$$
\begin{aligned}
u(x, 0) & =U_{0}, \quad v(x, 0)=0, \quad\left(D_{B} \frac{\partial \phi}{\partial y}\right)_{y=0}=-\left(\frac{D_{T}}{T} \frac{\partial T}{\partial y}\right)_{y=0}, \\
u(x, \infty) & =0, \quad T(x, \infty)=T_{\infty}\left(h=h_{\infty}\right), \quad \phi(x, \infty)=\phi_{\infty},
\end{aligned}
$$

where $\phi_{\infty}$ is the ambient nanoparticles concentration, $T_{\infty}$ is the ambient temperature, and at the surface of the plate the Stefan's flow has been considered which controls the concentration gradient by the temperature gradient. This is quite fortunate that such a complicated flow allows for an exact self-similar solution, which has already been justified by Avramenko et al. [16]. The similarity variables utilized by [16] and then [18] are given by

$$
\eta=y \sqrt{\frac{U_{0}}{\nu_{f} x}}, \quad \rho u=\rho_{\infty} U_{0} f^{\prime}(\eta), \quad \rho v=\frac{\rho_{\infty}}{2} \sqrt{\frac{U_{0} \nu_{f}}{x}}\left(\eta f^{\prime}-f\right), \quad h=h_{\infty} H(\eta), \quad \phi=\Phi(\eta),
$$

due to which the governing system (8)-(11) is transformed to an equivalent system of ordinary differential equation of the form

$$
\begin{aligned}
M f^{\prime \prime \prime}+ & {\left[\frac{\bar{\rho}}{2} f+\left(M^{\prime}-2 M \frac{R^{\prime}}{R}\right) \Phi^{\prime}\right] f^{\prime \prime}+\left[\Phi^{\prime 2}\left[2 M\left(\frac{R^{\prime}}{R}\right)^{2}-M^{\prime} \frac{R^{\prime}}{R}-M \frac{R^{\prime \prime}}{R}\right]-\frac{\bar{\rho}}{2} \frac{R^{\prime}}{R} f \Phi^{\prime}-M \Phi^{\prime \prime} \frac{R^{\prime}}{R}\right] f^{\prime}=0, } \\
K H^{\prime \prime}+ & H^{\prime}\left[\bar{\rho} \operatorname{Pr} f \frac{R C}{R}+\Phi^{\prime}\left(K^{\prime}+\frac{1}{L e}+2\left(\frac{R^{\prime}}{R}-\frac{R C^{\prime}}{R C}\right)\left(K+\frac{\bar{D}}{L e}\right)\right)\right] \\
& +\Phi^{\prime 2}\left[\bar{D}\left(\frac{R^{\prime}}{R}-\frac{R C^{\prime}}{R C}\right)^{2}+K\left(2 \frac{R C^{\prime 2}}{R C^{2}}-2 \frac{R^{\prime}}{R} \frac{R C^{\prime}}{R C}+\frac{R^{\prime \prime}}{R}-\frac{R C^{\prime \prime}}{R C}\right)\right] \\
& +\frac{\bar{D} H^{\prime 2}}{L e H}+H\left(\frac{R^{\prime}}{R}-\frac{R C^{\prime}}{R C}\right)\left[K \Phi^{\prime \prime}+\Phi^{\prime 2}\left(\frac{1}{L e}+K^{\prime}\right)\right]=0, \\
\Phi^{\prime \prime} & {\left[1+\bar{D}\left(\frac{R^{\prime}}{R}-\frac{R C^{\prime}}{R C}\right) H\right]+\Phi^{\prime}\left[\frac{H^{\prime}}{D_{B}}\left(\frac{D_{T}^{\prime}}{H}+D_{B}^{\prime}\right)+\frac{\bar{\rho}}{2 R} S c f\right]+\bar{D}\left(\frac{H^{\prime \prime}}{H}-\frac{H^{\prime 2}}{H^{2}}\right) } \\
& +\Phi^{\prime 2}\left[\left(\frac{R^{\prime}}{R}-\frac{R C^{\prime}}{R C}\right)\left(\frac{D_{B}^{\prime}}{D_{B}} H+\frac{D_{T}^{\prime}}{D_{B}}\right)+\bar{D}\left(\frac{R C^{\prime 2}}{R C^{2}}-\frac{R^{\prime 2}}{R^{2}}+\frac{R^{\prime \prime}}{R}-\frac{R C^{\prime \prime}}{R C}\right)\right]=0,
\end{aligned}
$$

where $R C(\phi)=(1-\phi)+\phi\left(\rho c_{p} / \rho c_{f}\right), \quad M(\phi)=(1-\phi)^{-2.5}, R(\phi)=(1-\phi)+\phi\left(\rho_{p} / \rho_{f}\right), \quad K(\phi)=\left[k_{p}+2 k_{f}+\right.$ $\left.2 \phi\left(k_{p}-k_{f}\right)\right] /\left[k_{p}+2 k_{f}-\phi\left(k_{p}-k_{f}\right)\right], \quad \bar{\rho}=\rho_{\infty} / \rho_{f}, \quad \operatorname{Pr}=\mu_{f} c_{f} / k_{f}, \quad S c[H(\eta)]=\mu_{f} / \rho_{f} D_{B}, \quad \operatorname{Le}[H(\eta)]=$ $(S c[H(\eta)] / \operatorname{Pr})\left(\rho_{f} c_{f} / \rho_{p} c_{p}\right), \quad \bar{D}=D_{T} / D_{B}$. In this system primes in the functions $f, H$, and $\Phi$ stand for derivative w.r.t. $\eta$ while $R^{\prime}, R C^{\prime}, K^{\prime}, D_{T}^{\prime}$ and $M^{\prime}$ mean derivative w.r.t. $\Phi$ and the prime in the function $D_{B}$ denotes its derivative w.r.t $H$. The self-similar form of respective boundary conditions read as

$$
\begin{aligned}
f(0) & =0, \quad f^{\prime}(0)=\frac{R\left(\phi_{w}\right)}{\bar{\rho}}, \quad H(0)=\frac{h_{w}}{h_{\infty}}, \quad\left(\frac{H^{\prime}}{H}\right)_{\eta=0}=\Phi^{\prime}(0)\left(\frac{R C^{\prime}}{R C}-\bar{D}\right), \\
f^{\prime}(\infty) & =0, \quad H(\infty)=1, \quad \Phi(\infty)=\phi_{\infty} .
\end{aligned}
$$

In energy Eq. (10), enthalpy is replaced by temperature to eliminate the additional parameter $h_{w} / h_{\infty}$ arising in Eq. (17). Also, we assume that the specific heat capacity in Eq. (10) is taken as constant $c_{\infty}$ instead of a function of nanoparticles concentration for mathematical simplification. Furthermore, it will be assumed that $D_{B}$ and $D_{T}$ are constant and the temperature $T_{\infty}$ arising in Eq. (10) and (11) is also constant. The self-similar form of (10) and (11) under these assumptions read as

$$
\begin{aligned}
K \Theta^{\prime \prime}+\Theta^{\prime}\left(\frac{\Phi^{\prime}}{L e}+K^{\prime} \Phi^{\prime}+\frac{\bar{\rho}}{2} \operatorname{Pr} f\right)+\frac{D}{L e} \Theta^{\prime 2} & =0, \\
\Phi^{\prime \prime}-\frac{\bar{\rho}}{2 R} S c f \Phi^{\prime}+D \Theta^{\prime \prime} & =0,
\end{aligned}
$$

where $\Theta(\eta)=\left(T-T_{w}\right) /\left(T_{\infty}-T_{w}\right), \operatorname{Pr}=\left(\mu_{f} c_{\infty} / k_{f}\right), L e=(S c / \operatorname{Pr})\left(\rho_{f} c_{\infty} / \rho_{p} c_{p}\right)$, and $D=\left(D_{T} / D_{B}\right)\left(\left[T_{\infty}-T_{w}\right] / T_{\infty}\right)$. The boundary conditions (17) therefore take the following form

$$
f(0)=0, \quad f^{\prime}(0)=\frac{R\left(\phi_{w}\right)}{\bar{\rho}}, \quad \Theta(0)=0, \quad D \Theta^{\prime}(0)+\Phi^{\prime}(0)=0, \quad f^{\prime}(\infty)=0, \quad \Theta(\infty)=1, \quad \Phi(\infty)=\phi_{\infty} .
$$




\section{Entropy generation}

According to second law of thermodynamics, the entropy of the system always increases for any irreversible process and is directly related to loss of available work (Gouy-Stodela theorem). Therefore, the improvement into the thermodynamic performance of an engineering system requires the inclusion of thermal irreversibility analysis into the thermodynamic analysis. Bejan [1] initiated the work in this field and explained, in the light of second law of thermodynamics, that the two main factors (the heat transfer due to temperature difference and the viscous dissipation) are responsible for entropy production in a fluid flow. For a viscous (pure/base) fluid the expression of entropy generation as given by Bejan [1] for a steady two-dimensional boundary layer flow after boundary-layer approximation reads as

$$
\begin{aligned}
& S_{G}=\left(S_{G}\right)_{f}+\left(S_{G}\right)_{T}, \\
& S_{G}=\frac{\mu}{T_{\infty}}\left(\frac{\partial u}{\partial y}\right)^{2}+\frac{k}{T_{\infty}^{2}}\left(\frac{\partial T}{\partial y}\right)^{2},
\end{aligned}
$$

where the first term is due to viscous dissipation and the second term is due to temperature gradient. For the case of nanofluid flow involving non-uniform concentration of nanoparticles the above expression gets modified to include the concentration gradients. Therefore, the local entropy generation rate $\left(S_{G}\right)$, in view of Buongiorno's model, is expressed as

$$
S_{G}=\frac{k}{T^{2}}(\nabla T)^{2}+\frac{1}{T} \Phi^{*}+\frac{\rho_{p} c_{p} D_{B}}{\phi^{2}} \nabla \phi \cdot \nabla \phi+\frac{\rho_{p} c_{p} D_{T}}{\phi^{2} T} \nabla T \cdot \nabla \phi+\frac{\rho_{p} c_{p} D_{B}}{\phi^{2} T} \nabla T . \nabla \phi+\frac{\rho_{p} c_{p} D_{T}}{T^{2}}(\nabla T)^{2},
$$

where

is the differential operator and

$$
\nabla=\frac{\partial}{\partial x} \hat{i}+\frac{\partial}{\partial y} \hat{j}
$$

$$
\Phi^{*}=\mu\left(2\left[\left\{\frac{\partial u}{\partial x}\right\}^{2}+\left\{\frac{\partial v}{\partial y}\right\}^{2}\right]+\left[\frac{\partial v}{\partial x}+\frac{\partial u}{\partial y}\right]^{2}\right)
$$

is is the viscous dissipation function in two dimensions. After boundary layer approximation, the above equation read as

$$
\begin{aligned}
S_{G} & =\frac{k}{T_{\infty}^{2}}\left(\frac{\partial T}{\partial y}\right)^{2}+\frac{1}{T_{\infty}}\left(\frac{\partial u}{\partial y}\right)^{2}+\frac{\rho_{p} c_{p} D_{B}}{\phi_{\infty}^{2}}\left(\frac{\partial \phi}{\partial y}\right)^{2} \\
& +\frac{\rho_{p} c_{p} D_{T}}{\phi_{\infty}^{2} T_{\infty}}\left(\frac{\partial T}{\partial y}\right)\left(\frac{\partial \phi}{\partial y}\right)+\frac{\rho_{p} c_{p} D_{B}}{\phi_{\infty}^{2} T_{\infty}}\left(\frac{\partial T}{\partial y}\right)\left(\frac{\partial \phi}{\partial y}\right)+\frac{\rho_{p} c_{p} D_{T}}{T_{\infty}^{2}}\left(\frac{\partial T}{\partial y}\right)^{2} .
\end{aligned}
$$

From the above equation it is noted that, the entropy generation not only depends upon the thermophysical properties of nanofluid but also depends upon the Brownian diffusion. Hence, Eq. (24) reflects the contribution of concentration gradients, which is directly associated with the consideration of non-homogenous modeling of nanofluid. This fact highlights the importance and provides justification for the consideration of this problem. In view of similarity variables (13), Eq. (24) also transforms as,

$$
\begin{aligned}
\frac{N_{s}}{R e_{x}} & =\frac{S_{G}}{S_{G_{0}}}=\left(k(\phi)+\frac{D}{L e \Omega_{T}}\right) \Theta^{\prime 2}+M(\phi) \frac{\bar{\rho}^{2}}{R^{4}} \frac{B r}{\Omega_{T}}\left[R f^{\prime \prime}-f^{\prime} R^{\prime} \Phi^{\prime}\right]^{2} \\
& +\left(\frac{D}{\Omega_{T}^{2} \phi_{\infty}^{2} L e}+\frac{1}{L e \Omega_{T}}\right) \Theta^{\prime} \Phi^{\prime}+\frac{1}{\Omega_{T}^{2} \phi_{\infty}^{2} L e} \Phi^{\prime 2}
\end{aligned}
$$

where $S_{G_{0}}=k_{f}\left(\Omega_{T} / x\right)^{2}, \Omega_{T}=\Delta T / T_{\infty}, B r=\mu_{f} U_{0}^{2} / k_{f} \Delta T$ are the characteristic entropy, the dimensionless temperature, and Brinkman number, respectively. In convective heat transfer problems, the dimensionless parameter (irreversible distribution ratio) $\phi^{*}$ is considered to quantify the involvement of heat transfer rate, viscous dissipation, and diffusivity in the production of entropy. For the current problem it is defined as

$$
\phi^{*}=\frac{M(\phi) \frac{\bar{\rho}^{2}}{R^{4}} \frac{B r}{\Omega_{T}}\left[R f^{\prime \prime}-f^{\prime} R^{\prime} \Phi^{\prime}\right]^{2}}{\left(k(\phi)+\frac{D}{L e \Omega_{T}}\right) \Theta^{\prime 2}+\left(\frac{D}{\Omega_{T}^{2} \phi_{\infty}^{2} L e}+\frac{1}{L e \Omega_{T}}\right) \Theta^{\prime} \Phi^{\prime}+\frac{1}{\Omega_{T}^{2} \phi_{\infty}^{2} L e} \Phi^{\prime 2}} .
$$

Equation (26) indicates that, for $0<\phi^{*}<1$ thermal irreversibility is dominant, while the irreversibility due to fluid viscosity and diffusivity are dominant for $\phi^{*}>1$. In this connection, the local Bejan number $(B e)$ in terms of irreversibility ratio has the following form

$$
B e=\frac{1}{1+\phi^{*}} \text {. }
$$


Since the concentration of nanoparticles is non-uniform in the base fluid. Due to this fact, to examine the overall production of entropy in the flow phenomenon, the average values are computed. The dimensionless average entropy generation number (total entropy generation) and average Bejan number can be calculated by integrating local entropy generation number and local Bejan number by using the following formulae:

$$
\begin{aligned}
& \bar{N}_{s}=\frac{1}{\forall} \int_{\forall} N_{s} d \forall, \\
& \bar{B} e=\frac{1}{\forall} \int_{\forall} B e d \forall
\end{aligned}
$$

where $\forall$ represents boundary layer thickness.

\section{Local and total entropy generation}

A built-in package of MATLAB software bvp4c has been implemented for the Eqs. (14), (18) and (19) with boundary conditions (20) to get a numerical solution. The impact of involved parameters $\phi_{\infty}, D, S c, B r$, and $\operatorname{Pr}$ on entropy generation rate and Bejan number are explored through Figs. 2-12. All the graphical results show that the moving plate surface is a substantial source of irreversibility production, and its impact on entropy production gradually vanishes out as one

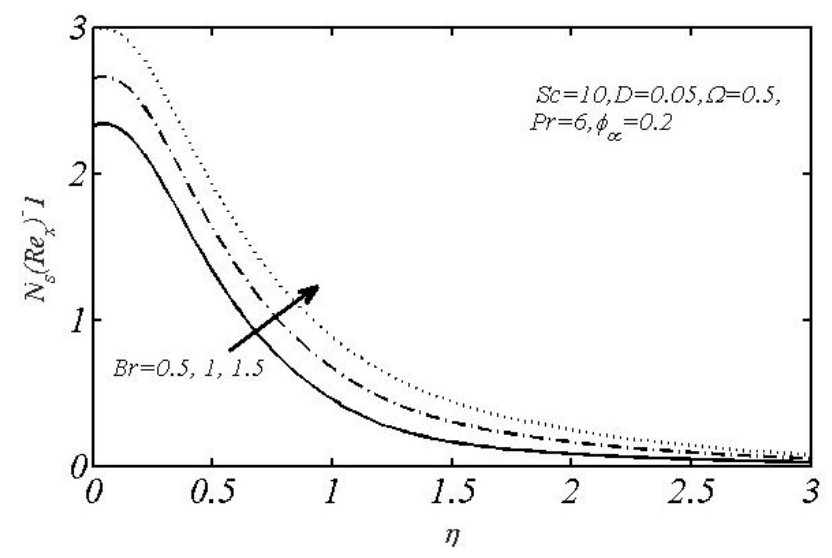

FIGURE 2. Local entropy generation profile for different Br.

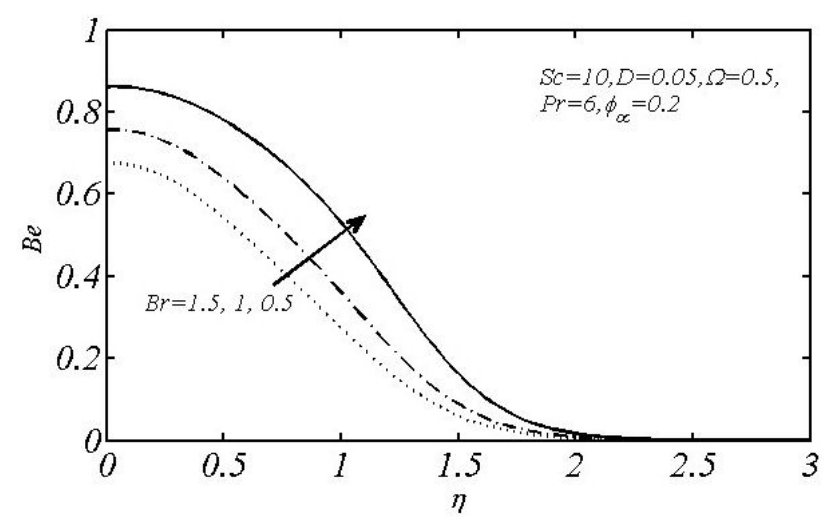

FIGURE 3. Be plotted against $\eta$ for different Brinkman number.

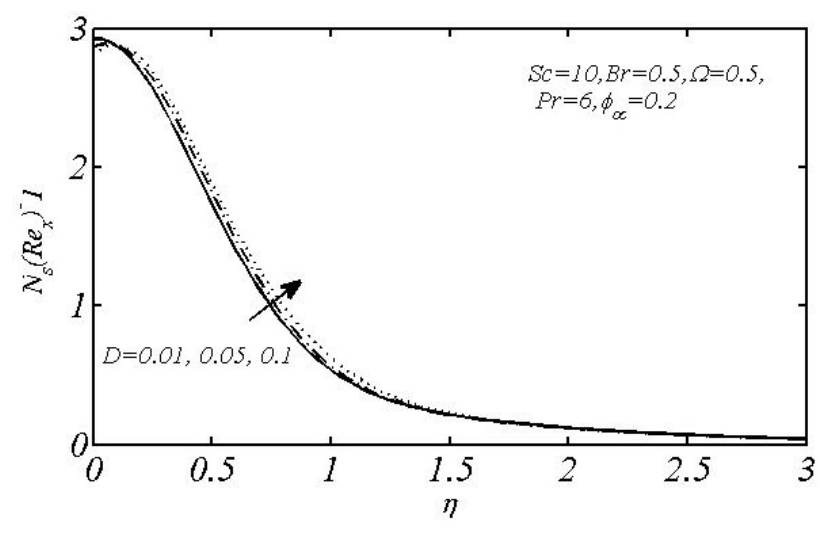

FIGURE 4. Dependence of local entropy generation number on $D$.

TABLE I. Average entropy number $\bar{N}_{s}$ and average Bejan number $\bar{B} e$ with variation of $B r$ at fixed $\operatorname{Pr}=6, \rho_{p} / \rho_{f}=3.98195$, $S c=10, \phi_{\infty}=0.1$ and $D=0.05$.

\begin{tabular}{cccccc}
\hline$B r$ & 0.5 & 1.0 & 1.5 & 2.0 & 3.0 \\
\hline $\bar{N}_{S}$ & 0.1566 & 0.1930 & 0.2294 & 0.2658 & 0.3386 \\
$\bar{B} e$ & 0.1016 & 0.0809 & 0.0685 & 0.0598 & 0.0481 \\
\hline
\end{tabular}

TABLE II. Impact of $D$ upon $\bar{N}_{s}$ and $\overline{B e}$ at fixed $\operatorname{Pr}=6$, $\rho_{p} / \rho_{f}=3.98195, S c=10, B r=0.5, \phi_{\infty}=0.1$

\begin{tabular}{ccccccc}
\hline$D$ & 0.01 & 0.05 & 0.1 & 0.3 & 0.5 & 0.6 \\
\hline $\bar{N}_{S}$ & 0.1534 & 0.1566 & 0.1594 & 0.1741 & 0.2016 & 0.2204 \\
$\bar{B} e$ & 0.0946 & 0.1016 & 0.1116 & 0.1341 & 0.1399 & 0.1445 \\
\hline
\end{tabular}

starts to get away from the plate. Finally, the entropy generation phenomenon completely vanishes in the free stream region. Figure 2 explores the impact of Brinkman number $(B r)$ on local entropy generation. This Figure depicts that the entropy generation increases as $B r$ takes larger values. This augmentation is quite obvious owing to fluid friction which corresponds to entropy production elevation. It is further observed that the $N_{s}$ profile also exhibits a boundary layer character for which the $N_{s}$-layer thickness increases by increasing the values of $\mathrm{Br}$. Figure 3 represents the effects of Brinkman number on local Bejan number $(\mathrm{Be})$. The local Bejan number decreases with the increase of $B r$. The higher values of $\mathrm{Br}$ correspond to boost the viscous effect which ultimately leads to fluid friction irreversibility dominance. However, for a fixed value of $\mathrm{Br}$, this Figure illustrates the dominance of thermal irreversibility at the heated surface but in distant region (far from boundary) the contribution of viscosity and diffusivity in the production of entropy becomes the major source. In this figure the $B e$-layer is seen 


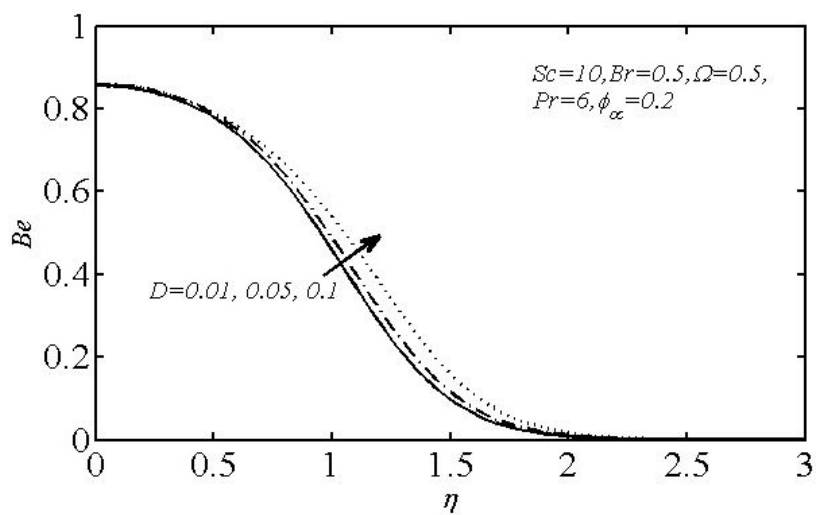

FIGURE 5. Impact of $D$ on local Bejan number.

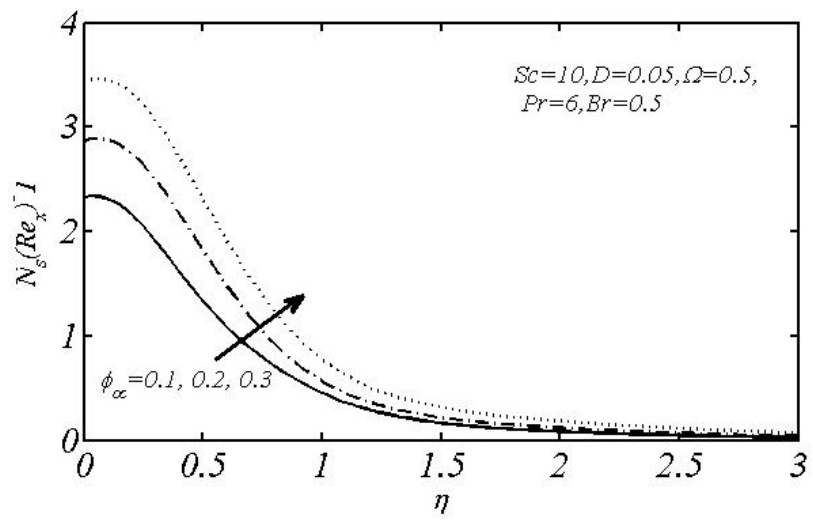

FIGURE 6. Variations in local entropy generation due to nanoparticle concentration parameter.

to not be affected significantly with an increase of $\mathrm{Br}$ values. The quantitative results of average entropy generation number $\left(\bar{N}_{s}\right)$ and average Bejan number $(\bar{B} e)$ with the variation of $\mathrm{Br}$ are reported in Table I in which average entropy generation shows an increasing trend for larger $\mathrm{Br}$ and a reverse trend for $(\bar{B} e)$. Figure 4 shows the effect of diffusion parameter $(D)$ on local entropy generation profile. This figure

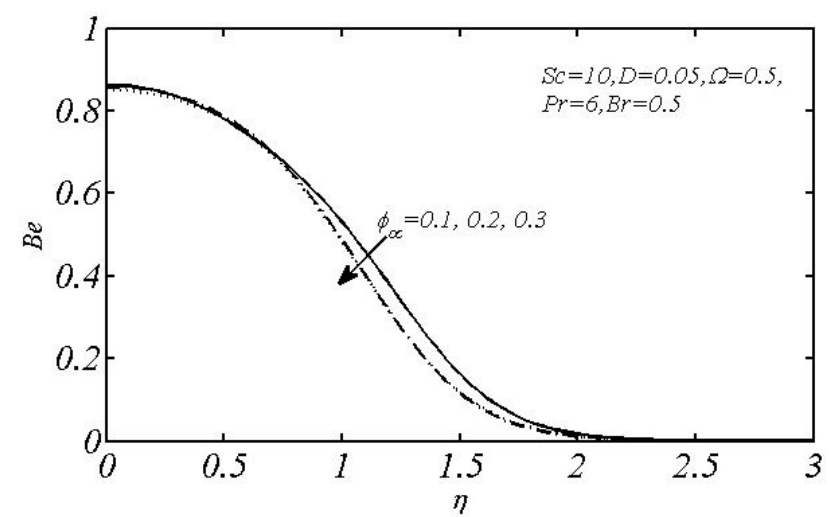

FIGURE 7. Local Bejan number plotted for different values of $\phi_{\infty}$.

reflects that the local entropy generation is increased near the moving plate upon increasing diffusion parameter $(D)$ and away from the wall the influence of diffusion parameter is reversed but such an influence is very less pronounced. Figure 5 also depicts a similar trend of local Bejan number $(B e)$ where it is plotted under the impact of diffusion parameter. The average Bejan number is also calculated and its variation with diffusion parameter is listed in Table II. Average Bejan number increases due to augmentation in diffusion parameter. But, for the considered range of diffusion parameter in Table II, it is observed that the average Bejan number attains lesser values than 0.5 which points out a very robust viscous irreversibility in the most part of boundary-layer region. The influence of nanoparticle concentration on $N_{s}$ is shown in Fig. 6 from where it can clearly be seen that loading of higher concentration corresponds to increase the entropy generation phenomenon which is, however, a trivial fact because the addition of nanoparticles in base fluid enhances the fluid friction and thermal conductivity. Impact of nanoparticles concentration $\phi_{\infty}$ on $B e$ is depicted in Fig. 7. Stronger $\phi_{\infty}$ leads to reduce the local Bejan number near the wall and in distant

TABLE III. Average values of $N_{s}$ and $B e$ for various values of $S c$ and $\phi_{\infty}$ at fixed $\operatorname{Pr}=6, B r=0.5, D=0.05$ and $\rho_{p} / \rho_{f}=3.98195$.

\begin{tabular}{|c|c|c|c|c|c|c|c|c|}
\hline$S c$ & & $\phi_{\infty}$ & & & & & & \\
\hline & & 0 & 0.03 & 0.05 & 0.07 & 0.1 & 0.15 & 0.2 \\
\hline \multirow[t]{2}{*}{10} & $\bar{N}_{S}$ & 0.1167 & 0.1187 & 0.1328 & 0.1431 & 0.1566 & 0.1784 & 0.2014 \\
\hline & $\bar{B} e$ & 0.0927 & 0.1179 & 0.1101 & 0.1053 & 0.1016 & 0.0995 & 0.0992 \\
\hline \multirow[t]{2}{*}{50} & $\bar{N}_{S}$ & 0.1167 & 0.1269 & 0.1356 & 0.1436 & 0.1554 & 0.1759 & 0.1981 \\
\hline & $\bar{B} e$ & 0.0927 & 0.0958 & 0.0952 & 0.0951 & 0.0952 & 0.0958 & 0.0967 \\
\hline \multirow[t]{2}{*}{80} & $\bar{N}_{S}$ & 0.1167 & 0.1274 & 0.1356 & 0.1433 & 0.1549 & 0.1753 & 0.1971 \\
\hline & $\bar{B} e$ & 0.0927 & 0.0948 & 0.0946 & 0.0946 & 0.0948 & 0.0995 & 0.0965 \\
\hline \multirow[t]{2}{*}{100} & $\bar{N}_{S}$ & 0.1167 & 0.1275 & 0.1355 & 0.1431 & 0.1546 & 0.1749 & 0.1969 \\
\hline & $\bar{B} e$ & 0.0927 & 0.0945 & 0.0944 & 0.0945 & 0.0947 & 0.0955 & 0.0965 \\
\hline \multirow[t]{2}{*}{1000} & $\bar{N}_{S}$ & 0.1167 & 0.1275 & 0.1347 & 0.1419 & 0.1532 & 0.1731 & 0.1948 \\
\hline & $\bar{B} e$ & 0.0927 & 0.0934 & 0.0934 & 0.0936 & 0.0940 & 0.0949 & 0.0960 \\
\hline
\end{tabular}




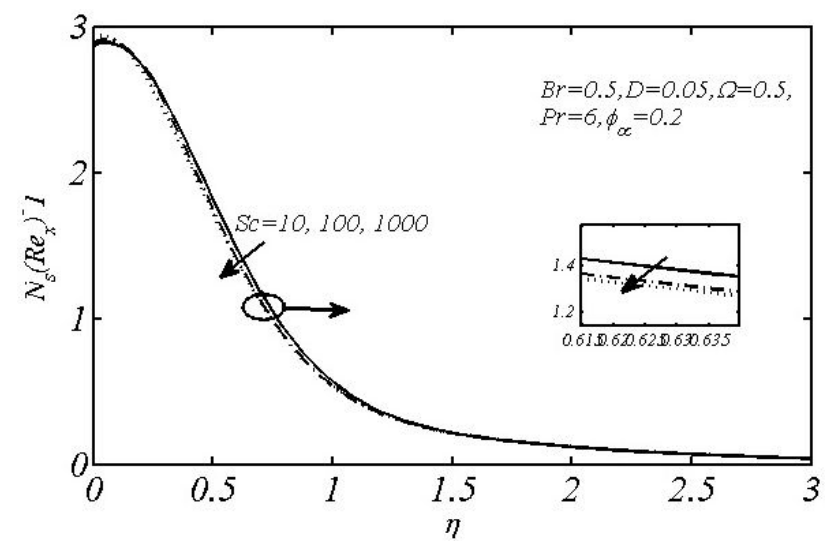

FIGURE 8. Effects of $S c$ on local entropy generation.

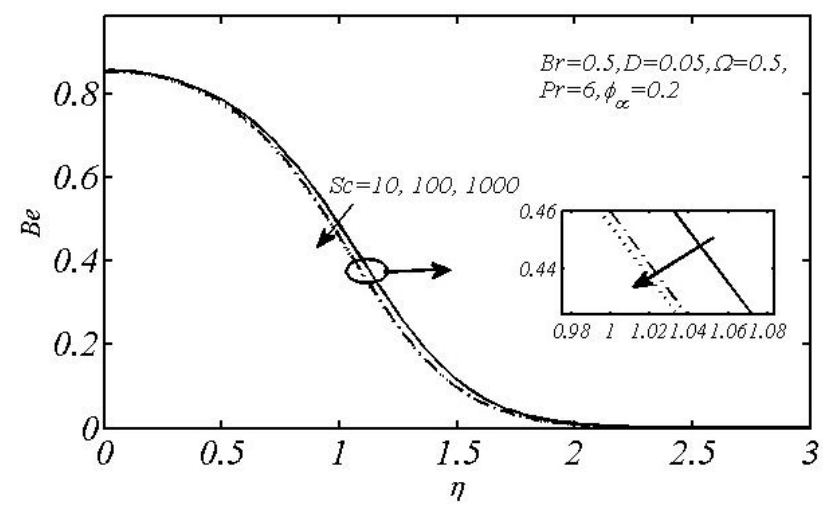

FIGURE 9. Local Bejan number profile for various values of $S c$.

region. The average entropy number along with average Bejan number have been summarized in Table III. Increasing the nanoparticle concentration from 0 to 0.2 , it is observed that, $\left(\bar{N}_{s}\right)$ is increased upto 1.72 times whereas $(\bar{B} e)$ is increased upto 1.07 times for $S c=10$. It is also interesting to note from this Table that, an increase in Schmidt number $(S c)$ leads to minimize the entropy generation. Bejan number also decreases with the variation of $S c$. For instance, in the case of $S c=1000$, for the considered range of nanoparticles concentration 1.67 and 1.03 times increment in $\left(\bar{N}_{s}\right)$ and $(\bar{B} e)$ is achieved, respectively. This means that loading of more and more nanoparticles leads to strengthen the heat transfer irreversibility within the boundary-layer. One can also notice from this Table that the total entropy generation number and Bejan number are increased up to almost $67 \%$ and $3 \%$, respectively, with $20 \%$ enhancement of $\phi_{\infty}$ in comparison to the pure fluid (Water) at $S c=1000$. Such a behavior of local entropy generation number and Bejan numbers can also be seen in Figs. 8 and 9. Influence of Prandtl number $(\mathrm{Pr})$ on the local entropy profile is explored in Fig. 10. For higher values of $P r$, intense enhancement in local entropy profile is noticed close to the boundary but again reverse effects are observed at some distance away from plate. Average of entropy number is increased to 1.56 times as Pr varies from 6 to 16 (refer to Table IV). Figure 11 depicts the behavior of local Bejan profile for various values of Pr. In the near wall region, the local Bejan number increases while reduces in the upper

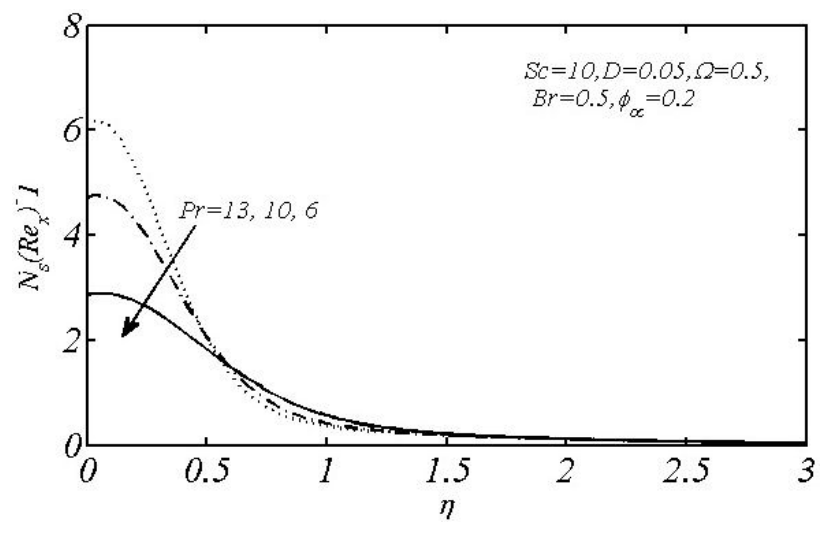

FIGURE 10. Local entropy generation profile for various values of $\operatorname{Pr}$.

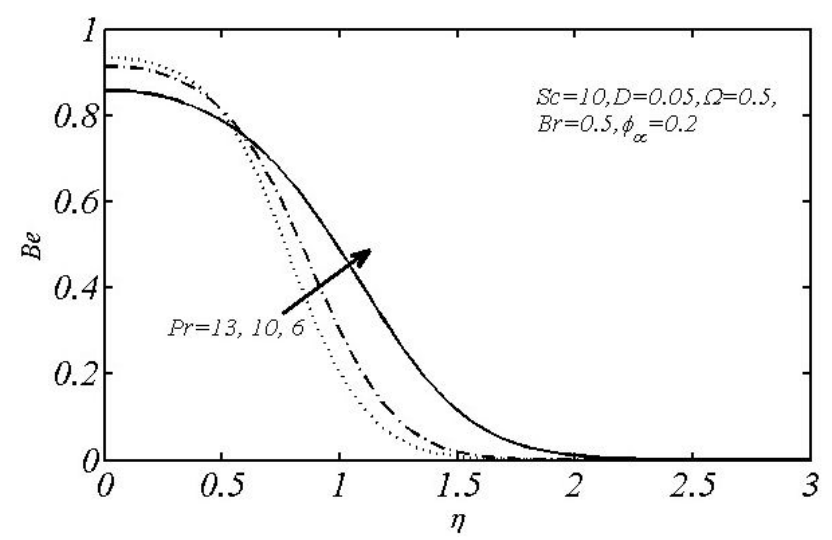

FIGURE 11. Influence of $\operatorname{Pr}$ on local Bejan number profile.

TABLE IV. Average entropy number $\bar{N}_{s}$ and average Bejan number $\bar{B} e$ for different base fluids at $D=0.05, \rho_{p} / \rho_{f}=3.98195$, $S c=10, \phi_{\infty}=0.1$ and $B r=0.05$.

\begin{tabular}{cccccc}
\hline $\operatorname{Pr}$ & 6 & 8 & 10 & 13 & 16 \\
$\bar{N}_{S}$ & 0.1567 & 0.1782 & 0.1970 & 0.2214 & 0.2434 \\
$\bar{B} e$ & 0.1001 & 0.0926 & 0.0876 & 0.0828 & 0.0795 \\
\hline
\end{tabular}

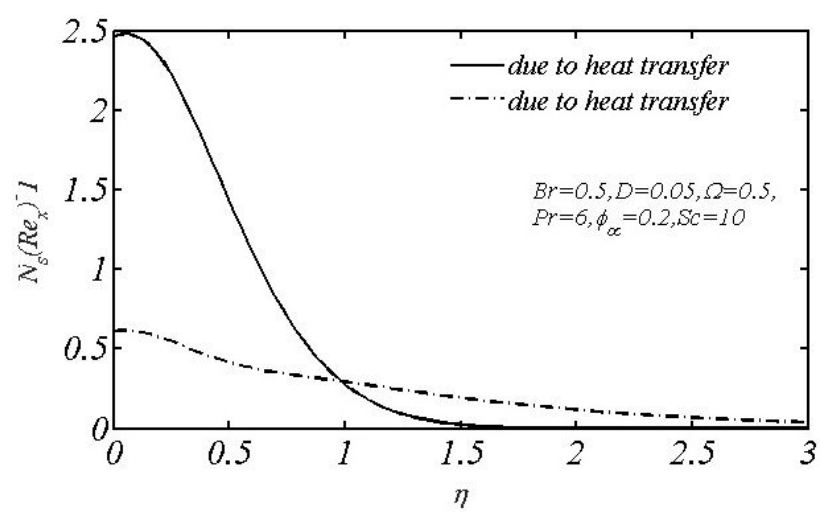

FIGURE 12. Comparative profile of entropy generation. 


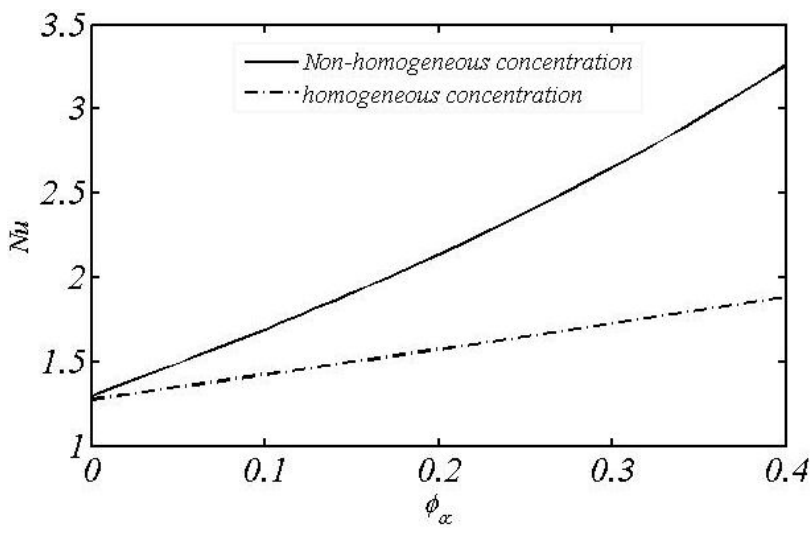

FIGURE 13. Nusselt number plotted against $\phi_{\infty}$.

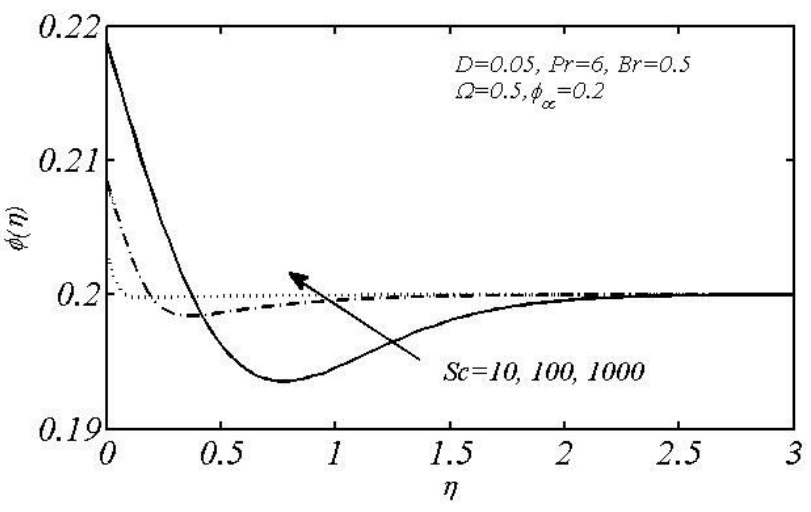

FIGURE 14. Concentration profile for different $S c$.

TABLE V. Comparison of Average values of entropy generation number and Bejan number for Non-homogenous concentration and homogenous concentration of nanoparticles at fixed $\operatorname{Pr}=0.6, \mathrm{Br}=0.5$, and $\rho_{p} / \rho_{f}=3.98195$.

\begin{tabular}{ccccc}
\hline$\phi_{\infty}$ & $\begin{array}{c}\bar{N}_{S} \text { (non-homogeneous) } \\
S c=10, D=0.05\end{array}$ & $\bar{N}_{S}$ (homogeneous) & $\begin{array}{c}\bar{B} e \text { (non-homogeneous) } \\
S c=10, D=0.05\end{array}$ & $\overline{B e}$ (homogeneous) \\
\hline 0.01 & 0.0966 & 0.1185 & 0.0860 & 0.0937 \\
0.05 & 0.1342 & 0.1255 & 0.1069 & 0.0981 \\
0.1 & 0.1567 & 0.1349 & 0.1001 & 0.1037 \\
0.2 & 0.2010 & 0.1558 & 0.0986 & 0.1153 \\
\hline
\end{tabular}

TABLE VI. Effects of $\phi_{\infty}$ on heat transfer rate for $\operatorname{Pr}=6, B r=0.5, \rho_{p} / \rho_{f}=3.98195$, and $D=0.05$.

$\begin{array}{ccccccccc}\phi_{\infty} & 0 & 0.01 & 0.03 & 0.05 & 0.07 & 0.1 & 0.15 & 0.2 \\ N u & 1.2754 & 1.3359 & 1.4120 & 1.4898 & 1.5693 & 1.6921 & 1.9072 & 2.1370\end{array}$

half of the times boundary layer with the increment of $\mathrm{Pr}$. It is seen from Table IV that, the reduction of average Bejan number is 0.8 times with the increment of $\mathrm{Pr}$. This behavior of Bejan number shows that irreversibility due to viscous dissipation starts to be dominant with an increment in $\mathrm{Pr}$. From Figs. 10 and 11 it is also noticeable that $N_{s}$-layer thickness and $\mathrm{Be}$ - layer thickness both decrease upon increasing the Prandtl number which is alike with the behavior of the temperature profile. A comparative analysis for average entropy generation number and average Bejan number for homogenous and non-homogenous concentration of nanoparticles in nanofluid has been given in Table V. It is observed that with $20 \%$ concentration of nanoparticles, the total entropy production is increased by almost $29 \%$ in the case of non-homogenous concentration in comparison with homogenous concentration. This clearly shows that the uniform distribution of nanoparticles in the mixture does underpredict the production of entropy. Influence of various physical parameters on heat transfer rate are illustrated through Tables VI-XIX. Impact of nanoparticles concentration on Nusselt number have been given in Table VI. The value of $N u$ is increased upto 1.67 times by the enhancement of $\phi_{\infty}$ from 0 to 0.2 .

This shows that heat transfer rate augments, which is a similar trend as the total entropy of the system increases by the increase of $\phi_{\infty}$ (see Table III). Moreover, in view of Tables II-IV and Tables VII-IX, it is seen that the similar impact of physical parameters $D, S c$, and $\operatorname{Pr}$ on average values of entropy generation and heat transfer rate has been noticed. This clearly reflects a strong relationship between the Nusselt number and the entropy generation number. Since, the heat is being transferred to the fluid, therefore, the entropy

TABLE VII. Impact of $D$ on heat transfer for $P r=6, B r=0.5$, $S c=10, \rho_{p} / \rho_{f}=3.98195, \phi_{\infty}=0.1$.

\begin{tabular}{ccccccc}
\hline$D$ & 0.01 & 0.05 & 0.1 & 0.3 & 0.5 & 0.6 \\
$N u$ & 1.6718 & 1.6921 & 1.7161 & 1.7973 & 1.8564 & 1.8786 \\
\hline
\end{tabular}

TABLE VIII. $N u$ for different base fluids at $D=0.05, B r=0.5$, $S c=10, \rho_{p} / \rho_{f}=3.98195, \phi_{\infty}=0.1$.

\begin{tabular}{cccccc}
\hline$P r$ & 6 & 8 & 10 & 13 & 16 \\
$N u$ & 1.6921 & 1.9839 & 2.2416 & 2.5847 & 2.8908 \\
\hline
\end{tabular}

TABLE IX. Impact of Schmidt number on $N u$ at $D=0.05$, $B r=0.5, \operatorname{Pr}=6, \rho_{p} / \rho_{f}=3.98195, \phi_{\infty}=0.1$.

\begin{tabular}{cccccc}
\hline$S c$ & 10 & 50 & 80 & 100 & 1000 \\
$N u$ & 1.6921 & 1.6752 & 1.6726 & 1.6716 & 1.6673 \\
\hline
\end{tabular}




\begin{tabular}{|c|c|c|c|c|c|c|c|c|}
\hline$S c$ & & $\phi_{\infty}$ & & & & & & \\
\hline \multirow{3}{*}{10} & & 0 & 0.03 & 0.05 & 0.07 & 0.1 & 0.15 & 0.2 \\
\hline & $\left(\bar{N}_{s}\right)_{\text {nano }} /\left(\bar{N}_{s}\right)_{\text {pure }}$ & 1 & 1.0171 & 1.1379 & 1.2262 & 1.3419 & 1.5287 & 1.7257 \\
\hline & $(\overline{B e})_{\text {nano }} /(\overline{B e})_{\text {pure }}$ & 1 & 1.2718 & 1.1877 & 1.1359 & 1.0960 & 1.0377 & 1.0701 \\
\hline \multirow[t]{2}{*}{50} & $\left(\bar{N}_{s}\right)_{\text {nano }} /\left(\bar{N}_{s}\right)_{\text {pure }}$ & 1 & 1.0874 & 1.1619 & 1.2305 & 1.3316 & 1.5072 & 1.6975 \\
\hline & $(\overline{B e})_{\text {nano }} /(\overline{B e})_{\text {pure }}$ & 1 & 1.0334 & 1.0269 & 1.0258 & 1.0269 & 1.0334 & 1.0431 \\
\hline \multirow[t]{2}{*}{80} & $\left(\bar{N}_{s}\right)_{\text {nano }} /\left(\bar{N}_{s}\right)_{\text {pure }}$ & 1 & 1.0916 & 1.1619 & 1.2279 & 1.3273 & 1.5012 & 1.6889 \\
\hline & $(\overline{B e})_{\text {nano }} /(\overline{B e})_{\text {pure }}$ & 1 & 1.0226 & 1.0204 & 1.0204 & 1.0226 & 1.0302 & 1.0409 \\
\hline \multirow[t]{2}{*}{100} & $\left(\bar{N}_{s}\right)_{n a n o} /\left(\bar{N}_{s}\right)_{\text {pure }}$ & 1 & 1.0925 & 1.1610 & 1.2262 & 1.3247 & 1.4987 & 1.6872 \\
\hline & $(\overline{B e})_{n a n o} /(\overline{B e})_{\text {pure }}$ & 1 & 1.0194 & 1.0183 & 1.0194 & 1.0215 & 1.0302 & 1.0409 \\
\hline \multirow[t]{2}{*}{1000} & $\left(\bar{N}_{s}\right)_{\text {nano }} /\left(\bar{N}_{s}\right)_{\text {pure }}$ & 1 & 1.0925 & 1.1542 & 1.2159 & 1.3127 & 1.4832 & 1.6692 \\
\hline & $(\overline{B e})_{\text {nano }} /(\overline{B e})_{\text {pure }}$ & 1 & 1.0075 & 1.0075 & 1.0097 & 1.0140 & 1.0237 & 1.0355 \\
\hline
\end{tabular}

of the fluid is increased by the increase of Nusselt number. The relative values of average entropy and Bejan number is calculated through Table $\mathrm{X}$ for different values of nanoparticle concentration and $S c$. It is observed that a $20 \%$ addition of nanoparticles in the working fluid increased up to $72 \%$ the entropy generation. Although addition of nanoparticles enhanced heat transfer rate, the results of Table $\mathrm{X}$ show a higher energy consumption. The impact of Schmidt number upon relative entropy generation is shown in Table X. It is noted that large values of $S c$ reduce entropy generation. It means that due to weak Brownian motion entropy production minimizes as well as the heat transfer rate also reduces. Figure 12 highlights the plot of separate entropies generated due to heat transfer and viscous dissipation. It is seen that in the vicinity of the plate entropy production due to heat transfer is higher than the entropy due to viscous dissipation and this behavior is reversed in a faraway region. Moreover, entropy production due to viscous dissipation is quite small as compared to the entropy produced due to heat transfer. Nusselt number as a function of $\phi_{\infty}$ is plotted in Fig. 13 for both homogeneous and non-homogeneous concentration of nanoparticles. Increase of $\phi_{\infty}$ augments heat transfer rate but this enhancement is higher in case of non-homogeneous concentration. Concentration profile for large values of $S c$ is observed through Fig. 14, in which it is clearly seen that the concentration of nanoparticles within the boundary layer is not homogeneous rather the concentration profile also possesses a boundary layer character. This justifies the consideration of current non-homogeneous nanofluid model.

\section{Concluding remarks}

The present analysis explores the entropy production phenomenon for a nanofluid flow with non-homogenous distribution of nanoparticles. On the basis of results of current study, it is concluded that
- Both the local and total entropy generation numbers increase with the increment of Brinkman number and diffusion parameter. Whereas a reverse trend is noticed in local and total Bejan number except from the fact that increase in diffusion parameter leads to increase the local Bejan number near the plate surface.

- Increase in nanoparticles concentration also results in an increment of both local and total entropy production within the boundary-layer region. In conclusion, adding more nanoparticles is in fact very useful as the fluid friction contribution in entropy production is tolerably less than the contribution of heat transfer. A similar trend is also observed for the average Bejan number.

- Almost 67\%, and 3\%, increase in total entropy generation, and Bejan number, respectively, is observed for the $20 \%$ concentration of nanoparticles in comparison to the pure fluid, i.e., water when Schmidt number is kept fixed at 1000 .

\section{Nomenclature}

$\begin{array}{ll}x, y & \text { Spatial coordinates } \\ u, v & \text { Velocity components } \\ c & \text { Specific heat of fluid } \\ h & \text { Enthalpy } \\ U_{0} & \text { Constant wall velocity } \\ T & \text { Temperature of the fluid } \\ D_{T} & \text { Thermophoretic diffusion coefficient } \\ D_{B} & \text { Brownian diffusion coefficient } \\ D & \text { Diffusion parameter } \\ d_{p} & \text { Nanoparticle diameter } \\ k_{B} & \text { Boltzman constant } \\ T_{\infty} & \text { Ambient temperature } \\ T_{w} & \text { Temperature at the surface }\end{array}$




$\begin{array}{ll}P r & \text { Prandtl number } \\ k & \text { Thermal conductivity of fluid } \\ S c & \text { Schmidt number } \\ B r & \text { Brinkman number } \\ S_{G} & \text { Volumetric rate of entropy generation } \\ S_{G_{0}} & \text { Characteristic entropy generation rate } \\ N_{s} & \text { Dimensionless entropy number } \\ B e & \text { Bejan number } \\ \bar{N}_{s} & \text { Average entropy generation } \\ \bar{B} e & \text { Average Bejan number } \\ \text { Greek Letters } & \\ \eta & \text { Similarity variable } \\ \Theta & \text { Dimensionless temperature }\end{array}$

$\begin{array}{ll}\mu & \text { Dynamic viscosity } \\ \nu & \text { Kinematic viscosity } \\ \rho & \text { Density of the fluid } \\ \beta & \text { Thermal expansion } \\ \Phi & \text { Dimensionless concentration } \\ \Phi^{*} & \text { Viscous dissipation function } \\ \phi & \text { Nanoparticle concentration } \\ \phi^{*} & \text { Irreversible distribution ratio } \\ \text { Subscripts } & \\ f & \text { Pure fluid } \\ p & \text { nanoparticles }\end{array}$

62 (2013) 515, https://doi.org/10.1016/j. ijheatmasstransfer.2013.03.004

A. Bejan, A study of entropy generation in fundamental convective heat transfer, J. Heat Transfer, 101 (1979) 718. https : //doi.org/10.1115/1.3451063

2. A. Bejan, Second law analysis in heat transfer, Energy, 5 (1980) 720, https://doi.org/10.1016/ 0360-5442(80) 90091-2.

3. A. Bejan, Entropy generation through heat and fluid flow, (Wiley, New York, 1982)

4. J.Y. San, W.M. Worek, and Z. Laven, Entropy generation in convective heat transfer and isothermal mass transfer, J. Heat Trans. 109 (1987) 647, https://doi.org/10. $1115 / 1.3248137$

5. B. Yilbas, Entropy analysis of concentric annuli with rotating outer cylinder, Int. J. Energy, 1 (2001) 60. https: //doi.org/10.1016/S1164-0235(01)00011-5.

6. S. Mahmud, and R.A. Fraser, The second law analysis in fundamental convective heat transfer problems Int. J. Therm. Sci. 42 (2003) 177. https://doi.org/ 10.1016/S1290-0729(02)00017-0

7. A.S. Butt, S. Munawar, A. Ali, and A. Mehmood, Entropy generation in the Blasius flow under thermal radiation, Phys. Scr. 85 (2012) 035008, https://doi.org/10. 1088/0031-8949/85/03/035008.

8. O. D. Makinde, Second law analysis for variable viscosity hydromagnetic boundary layer flow with thermal radiation and newtonian heating, Entropy 13 (2011) 1446, https : / / doi. org/10.3390/e13081446

9. A.S. Butt, S. Munawar, A. Ali, and A. Mehmood, Entropy generation in hydrodynamic slip flow over a vertical plate with convective boundary, J. Mech. Sci. Tech. 26 (2012) 2977, https://doi.org/10.1007/s12206-012-0701-3.

10. A. Mehmood, M.S. Iqbal, S. Khan, and S. Munawar, Entropy analysis in moving wavy surface boundary layer, Therm. Sci. 23 (2019) 233, https://doi.org/10. 2298/TSCI161029029M

11. M.M. Rashidi, S. Abelman, and N.F. Mehr, Entropy generation in steady MHD flow due to a rotating porous disk in a nanofluid, Int. J. Heat Mass Tran.
12. A. Noghrehabad, M.R. Saffarian, R. Pourrajab, and M. Ghalambaz, Entropy analysis for nanofluid flow over a stretching sheet in the presence of heat generation/absorption and //doi.org/10.1007/s12206-013-0104-0

13. M.M. Rashidi, M.M. Bhatti, M.A. Abbas, and M.E. Ali, Entropy generation on MHD blood flow of nanofluid due to peristaltic waves, Entropy 18 (2016) 117, https://doi.org/ $10.3390 / \mathrm{e} 18040117$

14. M. Almakki, S. Dey, S. Mondal, and P. Sibanda, On unsteady three-dimensional axisymmetric MHD nanofluid flow with entropy generation and thermo-diffusion effects on a non-linear stretching sheet, Entropy 19 (2017) 168, https://doi. org/10.3390/e19070168

15. S.E.B. Magia, C.T. Nugyen, N. Glanis, and G. Roy, Heat transfer behaviours of nanofluids in a uniformly heated tube, Supperlattices Microstruct. 35 (2004) 543, https://doi.org/ $10.1016 / j . s p m i .2003 .09 .012$

16. A.A. Avramenko, D.G. Blinov, and I.V. Shevchuk, Self-similar analysis of fluid flow and heat-mass transfer of nanofluids in boundary layer, Phys. Fluids 23 (2011) 082002, https: //doi.org/10.1063/1.3623432

17. A.A. Avramenko, D.G. Blinov, I.V. Shevchuk, and A.V. Kuznetsov, Symmetry analysis and self-similar forms of fluid flow and heat-mass transfer in turbulent boundary layer flow of a nanofluid, Phys. Fluids 24 (2012) 092003, https: //doi.org/10.1063/1.4753945

18. A. Mehmood, and M. Usman, Non-uniform nanoparticle concentration effects on moving plate boundary layer, Can. J. Phys. 94 (2016) 1222, https://doi.org/10. 1139/cjp-2016-0129

19. M. Frank, D. Anderson, E.R. Weeks, and J.F. Morris, Particle migration in pressure driven low of a Brownian suspension, J. Fluid Mech. 493 (2003) 363, https://doi.org/10. $1017 / \mathrm{S} 0022112003006001$ partial slip, J. Mech. Sci. Tech. 27 (2013) 927, https: 
20. D. Mehri, E. Lemaire, G. Bossis, and F. Moukalled, Particle migration in a concentrated suspension flowing between rotating parallel plates: investigation of diffusion flux coefficients, J. Rheol. 49 (2005) 1429, https: //doi.org/10.1122/ 1.2079247 .

21. Y.L. Ding, and D. Wen, Particle migration in a flow of nanoparticle suspensions, Powder Tech. 149 (2005) 84, https:// doi.org/10.1016/j.powtec.2004.11.012

22. J. Buongiorno, Convective transport in nano-fluids, ASME J. Heat Tran. 128 (2006) 240, https: //doi.org/10.1115/1.2150834

23. R.K. Tiwari, and M.K. Das, Heat transfer augmentation in a two-sided lid-driven differentially heated square cavity utilizing nanofluids, Int. J. Heat Mass Tran. 50 (2007) 2002, https://doi.org/10.1016/j. ijheatmasstransfer.2006.09.034

24. N. Dalir, M. Dehsara, and S.S. Nourazar, Entropy analysis for magnetohydrodynamic flow and heat transfer of a Jeffrey nanofluid over a stretching sheet, Energy 79 (2015) 351, https://doi.org/10.1016/j.energy.2014. 11.021

25. V. Bianco, O. Manca, S. Nardini, and K. Vafai, Heat Transfer Enhancement with Nanofluids (CRC Press, Boca Raton, 2015), https://doi.org/10.1201/b18324 